\title{
Psychoactive Substance Use among Medical/Health Faculty Undergraduate Students
}

\author{
P Whitehorne-Smith ${ }^{1}, \mathrm{C}$ Mitchell $^{2}$, WD Abel ${ }^{1}, \mathrm{~J} \mathrm{Harrison}^{1}$
}

\begin{abstract}
Objective: This study sought to explore the drug use practices of undergraduate students within the Faculty of Medical Sciences, The University of the West Indies, Mona, Jamaica.

Method: This study was a multicentre study carried out in Jamaica and six Latin American countries. The study utilized a cross-sectional design using a survey method of data collection. A list of compulsory classes for first-and second-year undergraduate students in the Faculty of Medical Sciences was retrieved by researchers and then cluster sampling was used to choose lectures to carry out data collection. The instrument utilized for the study was a self-report questionnaire which consisted of 58 questions which enquired about sociodemographic information, psychoactive substance use and associated consequences.

Results: A total of 380 students (78 males, 302 females) participated in the study; 115 (30.3\%) reported a past year prevalence of psychoactive substance use. Roughly half (50.8\%) reported that they first used substances when they were 15-19 years old. Students also reported a past month prevalence of alcohol use (16.6\%), prescription drugs without a prescription (4.5\%), tobacco (2.4\%) and cannabis (2.1\%) use.

Conclusion: These preliminary results on substance use patterns among students in the Faculty of Medical Sciences indicate urgent need for further research among this population. Such research should be used to inform prevention and treatment programmes that will directly target this student population.
\end{abstract}

Keywords: Medical students, substance use, university students

WIMJ Open 2015; 2 (1): 11

\section{INTRODUCTION}

The transition from high school to university can be a very stressful experience. The new experience for some students living on their own with reduced parental control - places them at heightened risk of substance use/abuse $(1,2)$. Also, university campus life is often a culture all to itself with numerous opportunities to experiment with various substances which students may not have been exposed to before. Such exposure comes at parties or "raves", both on campus and off. University culture also often promotes the heavy use of alcohol and other substances as stress relief and for fun (1).

Students of the Faculty of Medical Sciences are of particular concern to researchers, as they are involved in the helping professions, which may be more stressful and demanding than other areas of study. According to Maddux et al, many individuals (students and/or graduates) from medical sciences career are substance users/abusers. They found that from a sample of 133 medical students in the United States of America (USA), nearly all used alcohol,

From: ${ }^{1}$ Department of Community Health and Psychiatry, The University of the West Indies, Kingston 7, Jamaica and ${ }^{2}$ Faculty of Behaviour Science, International University of the Caribbean, Kingston 5, Jamaica.

Correspondence: Mrs P Whitehorne-Smith, Department of Community Health and Psychiatry, The University of the West Indies, Kingston 7, Jamaica. E-mail: patrice.whitehorne@yahoo.com
$57 \%$ used cannabis, $20 \%$ used cocaine and $40 \%$ used opioids (3).

McCabe et al noted that many medical students began using substances prior to medical school, with alcohol being the most frequently used substance. Some of the reasons cited for use were recreational, performance enhancement and self-medication for stress, anxiety and pain (4). Upegui, as quoted in Urrego, found that among medical students in Bogota, Colombia, stress, triggered by increased workload during the last year of internship, produced a significant increase in the incidence of anxiety symptoms, depression, somatic complaints, consumption of psychoactive substances and deterioration in the quality of life of some of these students (5).

Additionally, this group tends to be under-represented in studies of this nature and is not often thought of as substance users (3). These students need special attention as it relates to alcohol and psychoactive drug consumption, as they are the persons who impart health knowledge at the community level (6). The use of licit and/or illicit psychoactive substances by individuals in the medical science professions can be detrimental for others, as their responsibility and judgment as service providers may be compromised and result in harm to others. This study therefore sought to explore the drug use practices of undergraduate students within the Faculty of Medical Sciences, The University of the West Indies, Mona, Jamaica. 


\section{SUBJECTS AND METHODS}

This was a multicentre exploratory study which utilized a cross-sectional design using a survey method of data collection. The study was implemented in six Latin American countries and Jamaica. The researchers utilized a selfreport questionnaire which captured sociodemographic characteristics, substance use patterns, reasons for drug use, substance abuse, level of participation in campus life and consequences associated with substance use. The questionnaire consisted of four sections with 58 closed-ended questions which allowed respondents to provide additional information where necessary.

The sample size was calculated from the total of firstand second-year students of each medical sciences programme in each university using the "Sample Size Calculation Programme for Survey System" (7). This programme is based on a confidence interval of $95 \%$ significance and calculated on a lifetime prevalence of alcohol use in the general population within each country (8). This calculation revealed a minimum sample of 270 participants to be considered representative.

The inclusion criterion for the study was either fulltime or part-time students currently enrolled in either the first- or second-year programme in the Faculty of Medical Sciences of their universities. In all the participating universities, a 'Psychology' major was a part of their medical sciences faculties, so for consistency, students from this area of study were also included in this research.

A cluster sampling technique of data collection was used. Each university's list of compulsory courses for each programme in the Faculty of Health/Medical Sciences was gathered by the researchers for the first- and second-year programme levels. Each researcher randomly selected one or more compulsory classes, as necessary, to meet the sample size.

Permission to carry out the study was granted by the ethics committee of the participating university and by the principal of the university. Once permission was granted, particular lecturers in the medical sciences faculty were approached to allow researchers to gather information from their class during lecture time. Data were collected by trained research assistants after informed consent forms were signed and collected from participants.

\section{Data analysis}

Data were entered and cleaned in the Statistical Package for Social Sciences (SPSS). Descriptive and inferential statistics were performed on the data, including frequency distribution, means and standard deviations of the research sample, as well as Chi-squared analysis.

\section{RESULTS}

A total of 380 students participated in the study. The ages of the participants ranged from 17-52 years with the mean (SD) age being $22.27( \pm 5.67)$ years. Most participants were female $(79.5 \%)$ and a significant number was studying nursing $(49.7 \%)$. Also, the majority of the participants were in their first year of study (Table 1).

Table 1: Description of study participants

\begin{tabular}{lrr}
\hline Variables & $\mathbf{n}$ & $\mathbf{\%}$ \\
\hline Gender & & \\
Male & 78 & $20.5 \%$ \\
$\quad$ Female & 302 & $79.5 \%$ \\
University degree major & & \\
$\quad$ Medicine & 42 & $11.1 \%$ \\
$\quad$ Basic medicine: anatomy and physiology & 71 & $18.7 \%$ \\
Nursing & 189 & $49.7 \%$ \\
Pharmacy & 13 & $3.4 \%$ \\
Psychology & 62 & $16.3 \%$ \\
$\quad$ No response & 3 & $0.8 \%$ \\
Current year in university & & \\
$1^{\text {st }}$ year & 280 & $73.7 \%$ \\
$2^{\text {nd }}$ year & 97 & $25.5 \%$ \\
No response & 1 & $0.5 \%$ \\
\hline
\end{tabular}

$\mathrm{n}=380$

\section{Drug use patterns}

Thirty-five per cent of students admitted to using psychoactive substances in their lifetime and 30.3\% admitted past year use. There was no significant difference between males and females in terms of lifetime use or last year use ( $p$ $>0.05$, respectively). There was no significant difference between age or enrolment status and lifetime use or last year use ( $p>0.05$, respectively).

The most commonly used psychoactive substance was alcohol, both in the past year $(27.9 \%)$ as well as in the past month (16.6\%). This was followed by cannabis and misuse of prescription drugs (Fig. 1). The misuse of prescription drugs is taking prescription medication for reasons other than intended by a doctor, or at a higher dosage than intended, or taking someone else's medication. The psychoactive prescription drugs that these students reported using fell into the categories of opioid pain relievers (eg codeine), stimulants (eg Ritalin) or sedatives/tranquillizers (eg benzodiazepines).

Most students who reported lifetime use of psycho-

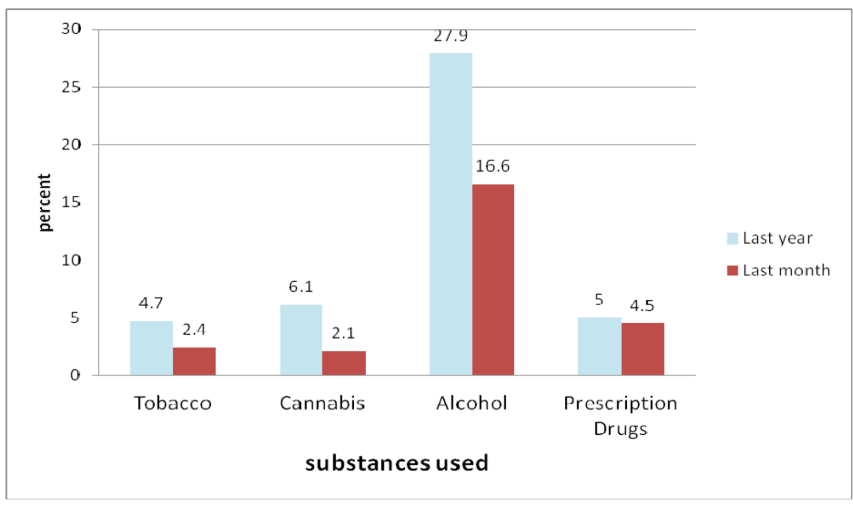

Fig. 1: Last year and last month use of psychoactive substances. 
active substances stated that their age at first use was 15-19 years $(50.8 \%)$, followed by $11-14$ years $(28 \%)$ and $0-10$ $(13.6 \%)$ years. A few persons reported first use between 20 and 24 years $(7.6 \%)$.

\section{Source of prescription drugs without a prescription}

Students were asked to identify ways in which prescription drugs are accessed without a prescription. The majority of persons gave no response or reported 'I don't know' (69.2\%). Of those who did respond (30.8\%), 'from a friend' (10.8\%) and 'from a friend's or relative's medicine cabinet' $(5.8 \%)$ were the most common sources (Fig. 2).

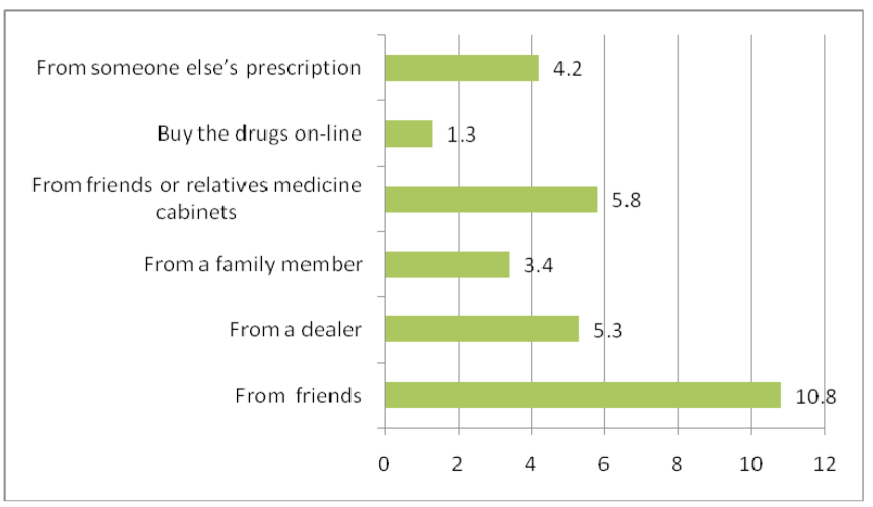

Fig. 2: Source of prescription drugs without a prescription.

\section{DISCUSSION}

The finding of the present study highlighted that alcohol was the most widely used substance. This finding supports numerous international research which found alcohol to be the leading substance of choice among students in tertiary educational institutions $(9,10)$. This finding is also in accordance with Jamaican drug use surveys which report alcohol, cannabis and tobacco as the psychoactive substance primarily used among Jamaican youth (11). Except in the case of the misuse of prescription drug use which was represented as the third most common drug used among this sample (5\% in the past year), this was different than what is generally reported among youth, where tobacco use and inhalant/solvent use typically follow after alcohol and cannabis use $(10,11)$. In this case, the possibility of greater access to prescription drugs among this population may be a factor in its place among the misused psychoactive substances.

Misuse of prescription drug is a fast growing area of concern among drug abuse experts. Research in the USA and Canada has found that one in four college students misuse prescription drugs (12-15). The reasons for prescription drug misuse have been linked to stimulants being considered a 'cognitive enhancer', as a means of self-medicating or to manage stress and anxiety; there is also the feeling that these substances pose less harm than other drugs, and there is greater accessibility to these substances by medical sciences students (12).
Despite this, the rates of misuse of prescription drugs were much lower than those reported by Maddux et al (3) who found percentages of $15 \%-40 \%$ across prescription drug categories. Prescription drug misuse has also increased over the past decade (14). The lower rates in Jamaica indicate that the problem may not yet be as extensive as it is in other countries, as well as the misuse may have been under-reported in this study.

Interestingly, half of the sample reported their age of first use of psychoactive substances at 15-19 years; this is different from the Jamaican population and youth surveys which indicate that most persons initiate drug use between 10 and 14 years $(8,11)$. It would be interesting to explore the reasons related to the later onset among medical students than in the wider youth population.

It is important to note the sources identified by students for obtaining these prescription drugs. These substances were garnered from friends, family members, friends/ relatives medicine cabinets, other persons' prescriptions or dealers. This finding suggests that there may be a general lack of awareness and appreciation of the dangers surrounding the misuse of prescription drugs.

By way of limitations, this study's only method of data collection was a self-administered questionnaire; it is likely that some respondents may have misrepresented information. Also, this study captured only a single faculty (except for psychology students). As such, no comparisons or generalizations can be made to undergraduate students in other disciplines.

\section{CONCLUSION}

In order to fully understand the severity of the substance abuse phenomenon at the participating university and other tertiary institutions, a study needs to be conducted on a larger scale, for the study findings to be generalized and to inform strategies that may be designed to address this chronic issue. There also needs to be networking with agencies in the position to assist those students who may need assistance with drug dependencies. Health promotion and education strategies targeting substance use are crucial to the whole process and need to be strengthened within the university.

\section{ACKNOWLEDGEMENTS}

This study was funded and supported by the Centre for Addiction and Mental Health (CAMH) and the InterAmerican Drug Abuse Commission (CICAD). Additionally, the following persons played a notable role in the original development of the multicentre study: Fabio Bautista Pérez, Andrés Herrera Rodríguez, Maria Do Perpétuo SS Nóbrega, Rosibel Prieto Cizel Ixbalanque Zea Iriarte Silva, Giselle Riquelme Hernández, Monica Veloza Gomez and Winston De La Haye. 


\section{REFERENCES}

1. Pillon S, O’ Brien B, Chavez K. The relationship between drugs use and risk behaviors in Brazilian university students. Rev Latino-am Enfermagem 2005; 13: 1169-76. Epub 2006 Feb 23.

2. Read J, Wood M, Davidoff O, McLacken J, Campbell J. Making the transition from high school to college: the role of alcohol-related social influence factors in student's drinking. Subst Abus 2002; 23: 53-65.

3. Maddux J, Hoppe S, Costello R. Psychoactive substance use among medical students. Am J Psychiatry 1986; 143: 187-91.

4. McCabe SE, West BT, Teter CJ, Boyd CJ. Trends in medical use, diversion, and nonmedical use of prescription medications among college students from 2003 to 2013: connecting the dots. Addict Behav 2014; 39: 1176-82. doi: 10.1016/j.addbeh.2014.03.008. Epub 2014 Mar 12.

5. Urrego D. Consumo de sustancias psicoactivas en estudiantes de especialidades médicas. Rev Salud Pública 2002; 4: 59-73.

6. Mesquita A, Henriette A, Castel S, Andrade A. Estudantes da Faculdade de Medicina da Universidade de São Paulo: uso de substâncias psicoativas em 1991. Rev Abp-Apal 1995; 17: 47-54.

7. Creative Research Systems. Sample size calculator. Petaluma, CA: Creative Research Systems; 2008 [cited 2008 Jul 28]. Available from: http://www.surveysystem.com/sscalc.htm

8. National Council on Drug Abuse. National Secondary School Survey 2006. Washington, DC: Organisation of American States/InterAmerican Drug Abuse Control Commission (OAS/CICAD); 2006.

9. Barrett S, Darredeau C, Pihl R. Patterns of simultaneous polysubstance use in drug using university students. Hum Psychopharmacol 2006; 21: 255-63.

10. Fiorini JE, Alves AL, Ferreira LR, Fiorini CM, Duraes SW, Santos RLD et al. Use of licit and illicit drugs at the University of Alfenas. Hosp Fac Med São Paulo 2003; 58: 199-206.
11. National Council on Drug Abuse. National Secondary School Survey 2013. Washington, DC: Organisation of American States/InterAmerican Drug Abuse Control Commission (OAS/CICAD); 2013.

12. McCabe SE, Teter CJ, Boyd CJ. Medical use, illicit use and diversion of prescription drugs. J Am Coll Health 2006; 54: 269-78.

13. McCabe SE, Cranford JA, Morales M, Young A. Simultaneous and concurrent polydrug use of alcohol and prescription drugs: prevalence, correlates, and consequences. J Study Alcohol 2006; 67: 529-37.

14. SAMHSA. Misuse of prescription drugs: data from the 2002, 2003, and 2004 National Surveys on Drug Use and Health. HHS Pub. No. (SMA) 06-4192. Rockville, MD: Office of Applied Studies; 2006.

15. Arria A, Wish E. Nonmedical use of prescription stimulants among college students. Pediatr Ann 2006; 35: 565-71.

Submitted 13 Oct 2014

Accepted 27 Oct 2014

Published 16 Mar 2015

Online: http://www.mona.uwi.edu/wimjopen/article/1625

(C) Whitehorne-Smith et al 2015

This is an open access article made freely available under Creative Commons Attribution 4.0 International (CC BY 4.0). Users are free to share, copy and adapt this work as long as the copyright holder (author) is appropriately and correctly credited. See http://creativecommons.org/ licences/by/4.0/deed.en_us for more information. 